\title{
EVALUATION AND ANALYSIS OF F-16XL WIND TUNNEL DATA FROM DYNAMIC TESTS
}

\author{
Sungwan Kim ${ }^{*}$ and Patrick C. Murphy ${ }^{\dagger}$ \\ NASA Langley Research Center \\ Hampton, Virginia 23681 \\ Vladislav Klein \\ The George Washington University \\ NASA Langley Research Center \\ Hampton, Virginia 23681
}

\begin{abstract}
$\underline{\text { Abstract }}$
A series of wind tunnel tests were conducted in the NASA Langley Research Center as part of an ongoing effort to develop and test mathematical models for aircraft rigid-body aerodynamics in nonlinear unsteady flight regimes. Analysis of measurement accuracy, especially for nonlinear dynamic systems that may exhibit complicated behaviors, is an essential component of this ongoing effort. In this paper, tools for harmonic analysis of dynamic data and assessing measurement accuracy are presented. A linear aerodynamic model is assumed that is appropriate for conventional forced-oscillation experiments, although more general models can be used with these tools. Application of the tools to experimental data is demonstrated and results indicate the levels of uncertainty in output measurements that can arise from experimental setup, calibration procedures, mechanical limitations, and input errors.
\end{abstract}

\section{Introduction}

A concern in any scientific experiment is the issue of obtaining sufficient measurement accuracy. In particular, it is a concern when investigating nonlinear dynamic systems since these systems can exhibit behaviors that may substantially complicate responses and model identification. For these experiments the amount of care taken in both experiment design and measurement procedures will be reflected in the final measurement accuracy. Two approaches can be used to estimate measurement accuracy. One approach is to use repeated measurements that allow a statistical estimate of the measurement variance to be made. This approach can be costly and time consuming in some cases; however, it has the advantage of directly reflecting measurement uncertainty. Another approach, based on system identification theory, is to utilize the difference between measured responses and that predicted by an adequate model. The advantage in this case is that repeated measurements are not required, although the presence of any modeling error will increase the residual error. Both approaches are presented and compared in this paper.

For the latter approach, a method is presented for harmonic analysis utilizing the least squares principle. In this approach, tools for assessing both accuracy of

\footnotetext{
${ }^{*}$ Research Engineer, Associate Fellow AIAA

${ }^{\dagger}$ Senior Research Engineer, Associate Fellow AIAA

*Professor Emeritus, Associate Fellow AIAA
}

measured outputs and accuracy of parameter estimates are presented. Application of the tools to both repeated and ensemble-averaged experimental data is demonstrated. In this analysis, a linear aerodynamic model appropriate for conventional forced-oscillation experiments is assumed although more general aerodynamic models can also be used with the tools presented.

In an effort to obtain a more general formulation of the aerodynamic model for aircraft, a series of wind tunnel tests were conducted in the NASA Langley Research Center (LaRC) 12-Foot Low-Speed Tunnel using a $10 \%$ scale model of the F-16XL. Two of tunnel tests, conducted in 1996 and 2000, provided static and dynamic data for nonlinear modeling research. Initial studies identifying unsteady aerodynamic models from these data were reported in Refs. [1]-[3]. During both the 1996 and 2000 experiments, for certain test conditions and signals, forced oscillation measurements did not reflect the expected harmonic motion related to the input signal. Single-run time histories and ensemble-averaged time histories were used to determine the final experimental results, namely, non-dimensional static forces, static moments, and stability derivatives. Combinations of stability derivatives were estimated in the conventional form of in-phase and out-of-phase components. No evaluation of the measurement data (before averaging) was done to test repeatability of the 1996 data. During the 2000 experiment, to reduce 
ensemble average errors and to enhance measurement accuracy analysis, each test was repeated 10 times. As part of a review process for these experiments, an initial evaluation of measurement accuracy for the 2000 experiment has been done and is presented in this paper.

Many problems that occur in testing can be quickly removed through careful monitoring by test engineers. However, in modern test facilities there is always a desire to automate systems to achieve greater production with fewer errors. This may tend to remove the test engineer from close observation of the experiment. Also, some measurement error may not be immediately observable without analysis. In any case, data with greater error than desired may be produced even when experimentalists exercise significant care. In this paper, examples of problem data that can occur during testing are provided and then procedures to reduce these problems, in an automated testing environment, are suggested.

The uncertainty of an experimental measurement is a combination of bias error and precision error. The squared sum of these terms is often used although other formulations are sometimes used ${ }^{4}$. In order to eliminate systematic errors or bias errors, careful calibration and attention to environmental variables are required. Bias errors can be difficult and in some cases impossible to estimate and remove from the data after the experiment is performed. Therefore in this paper only precision of the output measurements and estimated parameters will be evaluated. Also, no attempt is made to separate contributions to uncertainty from other factors such as scale effects or wind-tunnel turbulence. Since rigid body dynamics are assumed for this work and the dynamic rig is assumed to be rigid, aeroelastic or structural responses of the scale model and dynamic rig are not investigated.

In this paper, measured data are presented that have been obtained from single frequency forced-oscillation tests in the LaRC 12-Foot Tunnel. Analysis is limited to small amplitude dynamic data in order to limit nonlinear responses contained in the measurements. The approach for harmonic analysis described in this paper facilitates testing this assumption as part of the analysis.

\section{Model and Tests}

A three-dimensional view of the $10 \%$ scale F-16XL model is shown in Fig. 1. Dynamic tests were conducted in the LaRC 12-Foot Low-Speed Tunnel. For these tests, the model was mounted on a dynamic test rig through a six-component strain-gauge balance. The dynamic test rig is a computer controlled hydraulically actuated system that was sting-mounted on a C-strut support system. The mounting arrangement rotated the model about the reference center of gravity location of $0.558 \bar{c}$. Further description of the dynamic test rig may be found in Ref. [5].
The tests were conducted at a dynamic pressure of $192 \mathrm{~Pa} \mathrm{(=4} \mathrm{psf)} \mathrm{producing} \mathrm{an} \mathrm{equivalent} \mathrm{wind} \mathrm{speed}$ of $V=17.52 \mathrm{~m} / \mathrm{sec}$ and a Reynolds number of $10^{6}$ based on the mean aerodynamic chord. During dynamic tests, pitch angle readings were made with a Linear Variable Differential Transformer (LVDT), six-component force and moment data were obtained from a strain-gauge balance, and wind tunnel dynamic pressure measurements were obtained from pressure transducer.

For the 2000 experiment, data from singlefrequency forced oscillation in pitch were obtained at 5 different frequencies $(0.5,0.9,1.1,1.5$, and $2.0 \mathrm{~Hz})$ and 13 different values of the initial angle of attack $\left(\alpha_{0}=2,5,10,15,20,25,30,35,40,45,50,55\right.$, and 60 degrees) with amplitude of $\pm 5^{\circ}$. Repeated runs were made at the same test conditions and ensemble averages over the repeated runs were computed.

\section{Theoretical Tools}

Analysis and evaluation methods, metrics to assess measurement accuracy, and procedures that may help to identify and reduce measurement error are offered in this paper as a means of improving final experimental results. In this section, the main tools for analysis and evaluation are presented. First, a method for harmonic analysis that facilitates computation of higher harmonics and parameter error bounds is presented. This method effectively provides a test to detect nonlinear responses when nonzero high-order harmonics exist. Second, a conventional linear aerodynamic model representing a single degree of freedom forced-oscillation experiment is presented. Third, key metrics for assessing measurement accuracy are provided.

Measurement error, $e$, is generally assumed to be a zero mean, normally distributed, stochastic process with variance, $\sigma^{2}$. Measurement, $z$, true value, $y$, and error, $e$ are related as

$$
z=y+e .
$$

In this case, $E[z]=y$ and $E\left[(z-y)^{2}\right]=\sigma^{2}$. Since the true value, $y$, is unknown and unknowable, a model is usually identified that provides estimates of the responses as $\hat{y}$. Using the model estimates of response, the measurement equation becomes

$$
z=\hat{y}+\varepsilon .
$$

In this case, $E\left[\varepsilon^{2}\right]$ does not equal $\sigma^{2}$ since the model estimate $\hat{y}$ may contain error contributions from several sources. In addition to measurement error, the model may be inadequate to predict the responses. For example, if the inputs produce nonlinear responses a linear model will be inadequate and predict responses with additional errors. The analysis method suggested 
in this paper offers an approach that can detect this problem.

\section{Harmonic Analysis}

It is assumed that a periodic function $f(t)=f(t+2 \pi)$ with the period $2 \pi$ is specified by a time series of measured data points $z(0), z(1), \ldots z(N-1), z(N)=\mathrm{z}(0)$ at $t_{\mathrm{i}}=\frac{2 \pi}{N} i$ for $i=0,1, \ldots,(N-1)$. Further, the postulated model for $y(i)$ is assumed in the form

$$
y(i)=a_{0}+\sum_{k=1}^{m} a_{k} \cos \left(k \omega_{0} i\right)+\sum_{k=1}^{m} b_{k} \sin \left(k \omega_{0} i\right)
$$

where $\omega_{0}=\frac{2 \pi}{N}$ and $i=1,2, \ldots, N$ for $k=1,2, \ldots, m$.

The parameters $a_{0}, a_{k}$, and $b_{k}$ are the Fourier coefficients obtained from the measured data by applying the least squares principle

$$
\operatorname{Min} \sum_{i=1}^{N}[z(i)-y(i)]^{2} .
$$

The resulting estimates of the parameters are the solution to the equation

$$
\hat{\theta}=M^{-1} g
$$

where

$$
\begin{aligned}
& \hat{\theta}=\left[\begin{array}{llllllllll}
\hat{a}_{0} & \hat{a}_{1} & \hat{a}_{2} & \cdots & \hat{a}_{m} & \hat{b}_{1} & \hat{b}_{2} & \hat{b}_{3} & \cdots & \hat{b}_{m}
\end{array}\right]^{T} \\
& g=\left[\begin{array}{lll}
\sum_{i} z(i) & \sum_{i} z(i) \cos \left(\omega_{0} i\right) & \cdots \\
\sum_{i} z(i) \cos \left(m \omega_{0} i\right)
\end{array}\right. \\
& \left.\sum_{i} z(i) \sin \left(\omega_{0} i\right) \cdots \sum_{i} z(i) \sin \left(m \omega_{0} i\right)\right]^{T} \\
& M=\operatorname{diag}\left[\begin{array}{lllll}
N & \frac{N}{2} & \frac{N}{2} & \cdots & \frac{N}{2}
\end{array}\right] .
\end{aligned}
$$

Then the parameter estimates are obtained as

$$
\begin{aligned}
& \hat{a}_{0}=\frac{1}{N} \sum_{i} z(i) \\
& \hat{a}_{k}=\frac{2}{N} \sum_{i} z(i) \cos \left(k w_{0} i\right) \\
& \hat{b}_{k}=\frac{2}{N} \sum_{i} z(i) \sin \left(k w_{0} i\right)
\end{aligned}
$$

If the measurement errors $e(i)=z(i)-y(i)$ form a zero mean random sequence with the variance $\sigma^{2}$, then the parameter covariance matrix is

$$
\operatorname{Cov}(\hat{\theta})=\sigma^{2} M^{-1} \text {. }
$$

The estimation for the variance $\sigma^{2}$ can be found as

$$
s^{2}=\frac{1}{N} \sum_{i}[z(i)-\hat{y}(i)]^{2}
$$

where

$$
\hat{y}(i)=\hat{a}_{0}+\sum_{k} \hat{a}_{k} \cos \left(k \omega_{0} i\right)+\sum_{k} \hat{b}_{k} \sin \left(k \omega_{0} i\right) .
$$

From (6) and (7), the estimates of parameter variance are

$$
s^{2}\left(\hat{a}_{0}\right)=\frac{s^{2}}{N}, s^{2}\left(\hat{a}_{k}\right)=s^{2}\left(\hat{b}_{k}\right)=\frac{2 s^{2}}{N}
$$

for all $k$.

The model adequacy can be assessed by the Multiple Correlation Coefficient defined as

$$
R^{2}=1-\frac{\sum_{i}[z(i)-\hat{y}(i)]^{2}}{\sum_{i}[z(i)-\bar{z}]^{2}}, 0<R^{2}<1
$$

where $\bar{z}$ is the mean value of $z$.

\section{Conventional Forced Oscillation Analysis}

During conventional forced-oscillation tests the model is assumed to undergo periodic motion. In this study only small amplitude motions are considered so linear responses are expected. For analysis of this conventional oscillatory data it is assumed that the aerodynamic coefficients are linear functions of angle of attack, pitching velocity and their rates. Then the increment in the lift coefficient with respect to its mean value can be formulated as

$$
\Delta C_{L}=C_{L_{\alpha}} \Delta \alpha+\frac{\ell}{V} C_{L_{\dot{\alpha}}} \dot{\alpha}+\frac{\ell}{V} C_{L_{q}} q+\left(\frac{\ell}{V}\right)^{2} C_{L_{\dot{q}}} \dot{q}
$$

where for the harmonic motion

$$
\begin{aligned}
& \Delta \alpha=\alpha_{A} \sin \omega t \\
& \dot{\alpha}=q=\omega \alpha_{A} \cos \omega t \\
& \ddot{\alpha}=\dot{q}=-\omega^{2} \alpha_{A} \sin \omega t
\end{aligned}
$$

Substitution of (12) into (11) yields

$$
\begin{aligned}
\Delta C_{L} & =\alpha_{A}\left(C_{L_{\alpha}}-k^{2} C_{L_{\dot{q}}}\right) \sin \omega t+\alpha_{A} k\left(C_{L_{\dot{\alpha}}}+C_{L_{q}}\right) \cos \omega t \\
& =\alpha_{A}\left(\bar{C}_{L_{\alpha}} \sin \omega t+k \bar{C}_{L_{q}} \cos \omega t\right)
\end{aligned}
$$

where

and

$$
k=\omega \ell / V
$$

$$
\begin{aligned}
& \alpha_{A} \bar{C}_{L_{\alpha}}=\alpha_{A}\left(C_{L_{\alpha}}-k^{2} C_{L_{\dot{q}}}\right) \\
& \alpha_{A} k \bar{C}_{L_{q}}=\alpha_{A} k\left(C_{L_{\dot{\alpha}}}+C_{L_{q}}\right)
\end{aligned}
$$

represent the Fourier coefficients. The in-phase and out-of-phase components of $C_{L}\left(\bar{C}_{L_{\alpha}}\right.$ and $\left.\bar{C}_{L_{q}}\right)$ can be obtained by integrating the time histories of $\Delta C_{L}$ over $n_{c}$ cycles as

$$
\begin{aligned}
& \bar{C}_{L_{\alpha}}=\frac{2}{\alpha_{A} n_{c} T} \int_{0}^{n_{c} T} \Delta C_{L}(t) \sin \omega t d t \\
& \bar{C}_{L_{q}}=\frac{2}{\alpha_{A} k n_{c} T} \int_{0}^{n_{c} T} \Delta C_{L}(t) \cos \omega t d t
\end{aligned}
$$

where $T=2 \pi / \omega$. Using the results from harmonic analysis, the integrals in (17) represent continuous- 
time versions of (5). Therefore, the in-phase and out-ofphase components can be also expressed as

$$
\bar{C}_{L_{\alpha}}=\frac{\hat{b}_{1}}{\alpha_{A}}, \bar{C}_{L_{q}}=\frac{\hat{a}_{1}}{k \alpha_{A}}
$$

and their variance as

$$
s^{2}\left(\bar{C}_{L_{\alpha}}\right)=\frac{2}{N} \frac{s^{2}}{\alpha_{A}^{2}}, s^{2}\left(\bar{C}_{L_{q}}\right)=\frac{2}{N} \frac{s^{2}}{\left(k \alpha_{A}\right)^{2}} .
$$

\section{Evaluation Metrics and Outlier Rejection Rules}

The Multiple Correlation Coefficient $\left(R^{2}\right)$, expressed in (10), may be computed as part of the parameter estimation process. This metric varies between 0 and 1 and provides a measure of model adequacy. As the output signal fails to be represented by the model, the $R^{2}$ term will be reduced from 1 .

Mean square error $\left(s^{2}\right)$ defined in (7) characterizes the fit error of the model. A check to determine how well this metric reflects measurement accuracy can be made if repeated measurements are available. Calculation of the variance from repeated measurements provides a direct estimate of measurement accuracy. The variance can be computed from repeated measurements at each point in time as

$$
s_{i}^{2}\left(C_{a}\left(t_{i}\right)\right)=\frac{1}{N_{r}-1} \sum_{r=1}^{N_{r}}\left(\left[C_{a}\left(t_{i}\right)\right]_{r}-\bar{C}_{a}\left(t_{i}\right)\right)^{2}
$$

where $N_{r}$ is the number of repeated runs, $C_{a}\left(t_{i}\right)$ is a measured value of an aerodynamic coefficient at time $t_{i}$, and $\bar{C}_{a}$ is the mean value of $C_{a}$ for the repeated runs.

From these results an equivalent fit error, comparable to (7), can be computed as

$$
s^{2}=\frac{1}{N} \sum_{i} s_{i}^{2} .
$$

Comparison of $s^{2}$, computed from harmonic analysis (7), with the variance computed from repeated measurements (20)-(21) provides an indication of the level of measurement accuracy and an indication of good model adequacy if the two metrics are close in value.

During execution of an experiment, occasionally data points are obtained that appear to be outliers. If the experimentalist can determine verifiable problems with those points, then clearly these points should be removed. However, in some cases the results are not clearly wrong and no obvious problem can be found. Points outside the $2 \sigma$ bound might be good candidates that should be checked for problems. Statistical methods to check results can be easily automated so these tests can be used frequently. Among the various statistical methods, Chauvenet's Criterion ${ }^{4}$ is used in this paper.

\section{Data Evaluation and Analysis}

Evaluation and analysis methods proposed in this study are suggested as a method to assess measurement accuracy. In this paper, the methodology has been applied as part of a data post-processing procedure; however, some calculations may be done during the experiment for a faster on-line assessment. This section demonstrates application of the analysis methods to sample data from the 2000 experiment.

\section{Timing Signal}

The first data check for dynamic tests should be to determine the accuracy of the time stamp and then the sample time fidelity. Accuracy of the time stamp may be an important issue when a large number of signals are measured. Time stamp accuracy was not an issue for this study. Checking fidelity of the sample time during each duty cycle is a key test for dynamic experiments, especially when the analysis involves mathematical transforms. Commonly used transform algorithms require the sample time to be in equal time intervals. If a personal computer (PC) or workstation is used to run the real-time experiment, it is not uncommon for the operating system to introduce delays (from multitasking) in the duty cycle and thus produce uneven sample time intervals. If this type of error exists, it may require the data to be re-sampled or the experiment re-run depending on the degree of error. Re-sampling the data assumes that in spite of the duty cycle error, the measurements and the associated time stamp are still correct. If large delays or variable delays exist between the time stamp and measurement, then clearly no analysis would be possible. Fidelity of the sample time and accuracy of the time stamp must be ensured before any analysis is done.

A simple test metric to assess the degree of this problem is to compute the time difference, $\delta t_{a}$, between samples $n$ and $n+1 . \delta t_{a}$ is defined as

$$
\delta t_{a}=t_{n+1}^{a}-t_{n}^{a}
$$

where " $a$ " indicates actual sample times. For $100 \mathrm{~Hz}$ sample rate, a graph of $\delta t_{a}$ vs. sample number index should always equal 0.01 seconds for perfect sample timing. Another helpful sample-time metric is defined as the time difference between actual measured time and expected clock time or simulated time, both at sample $n . \delta t_{s}$ is defined as

$$
\delta t_{s}=t_{n}^{a}-t_{n}^{s}
$$

where " $s$ " indicates expected clock time or simulated time. This metric reflects the cumulative delay experienced during the run. Consequently, as delays occur this metric will grow in magnitude and indicate the total delay for the whole run at the last index value.

These two metrics (22) and (23) could be easily added to testing software to ensure high-fidelity sample times. 
An example highlights some of the issues associated with checking sample time fidelity. Analysis of the 2000 data revealed some cases where sampling was not occurring at $100 \mathrm{~Hz}$ as expected. As desktop computers and workstations have become very fast it has become popular to use these machines to run experiments and real-time simulations. Although the operating system was required to give the highest priority to the experimental task, duty cycle slips likely occurred due to multi-user, multi-tasking, or system level service. Fig. 2 (a) depicts the proper timing signal and duty cycle and Fig. 2 (b) depicts the timing problem when a duty cycle slip or frame overrun occurs. Fig. 2 (b) also shows another anomaly, unique to this experiment, i.e., sampling could be done faster than $100 \mathrm{~Hz}$ to compensate for any delay caused by duty cycle slips. This occurred because the data acquisition logic required the number of samples to be consistent with the desired sample rate and length of run where no sampling delays occur. Also the timing logic comparing actual time with desired time was not a controlling factor after a duty cycle slip.

Data, typical of $75 \%$ of all cases in this study, are shown in Fig. 3 (s726: $1.5 \mathrm{~Hz}$ and $\alpha_{0}=10^{\circ}$ ). In the figure, the pitch angle position measurement in the wind tunnel (equivalent to angle of attack) is analyzed and found to have numerous cases where the sample time is not stepping in increments of 0.01 seconds as expected. In this example, the $\delta t_{a}$ error is occasionally in the $20 \%$ to $30 \%$ range or 0.002 to 0.003 seconds. Note that no visible error appears in the angle of attack time history. Fig. 3 also shows another timing metric, $\delta t_{s}$. In this particular experiment, the timing logic requiring a faster sample rate to make up missed samples and logic requiring the system to "catch up" has the effect of canceling any delays that occur. Consequently only for this particular experiment does this metric return to zero after a delay period.

Fig. 4 shows a test case (s768: $1.5 \mathrm{~Hz}$ and $\alpha_{0}=35^{\circ}$ ) with timing slips greater than 6 samples. The angle-ofattack time history reflects this delay and shows significant distortion from the expected sinusoidal shape. $10 \%$ of all test runs contained timing delay greater than 0.05 seconds ( 5 cycle slips). In this case, the abnormality is clearly visible and easily detected and removed with only visual inspection.

To investigate the impact of duty cycle slip on experimental results a fictitious time delay followed by a catch-up sequence was added to good data. This created a second data set for comparison of in-phase and out-of phase coefficients. The test case used $1.5 \mathrm{~Hz}$ forced oscillation data at $\alpha_{0}=10^{\circ}$. Using ensemble-averaged data, estimates of mean values and standard deviations for this case are given in the below table.

\begin{tabular}{|c|c|c|}
\hline & Mean & Standard Error \\
\hline $\bar{C}_{N_{\alpha}}$ & 2.7273 & 0.0200 \\
\hline $\bar{C}_{N_{q}}$ & 4.5606 & 0.0582 \\
\hline
\end{tabular}

Using a single run from the ensemble of runs, fictitious data were created with varying time delay. Results shown in the table below provide mean values for a varying number of sample delays. The first column shows the mean value for the case without any delay.

\begin{tabular}{|c|c|c|c|c|c||}
\hline No. of Slips & 0 & 5 & 10 & 20 & 30 \\
\hline Delay (sec) & 0.00 & 0.05 & 0.10 & 0.20 & 0.30 \\
\hline $\bar{C}_{N_{\alpha}}$ & 2.7349 & 2.7314 & 2.7252 & 2.7347 & 2.7441 \\
\hline $\bar{C}_{N_{q}}$ & 4.5087 & 4.4944 & 4.4862 & 4.4391 & 4.0176 \\
\hline
\end{tabular}

Based on these results, estimated coefficients were within $\pm 2 \sigma$ of their correct mean values when delays were less than 10 samples. Since $90 \%$ of all test runs contained delays less than 0.05 seconds or 5 cycle slips, it is likely both in-phase and out-of phase coefficients for the 2000 experiment are computed within an error bound less than $\pm 2 \sigma$. This is consistent with the intended purpose of the 2000 experiment but may not be satisfactory for investigations where nonlinear dynamics play a significant role.

\section{Input Measurements}

Input tests involve calculation of test metric values that should bring attention to faulty input signals and errors that may not be immediately visible in a graphic representation or in a large data set generated in an automated fashion. Graphical tests are always included, however, to catch relatively large or obvious errors. The numerical values of the test metrics are generally very large for cases where the errors become visible in a graph.

The primary input signal for this study was angle of attack and it is the only input signal tested in this paper. Secondary inputs are temperature and tunnel dynamic pressure that had very slow variation and are assumed to be constant for these dynamic tests.

Three issues associated with the primary input measurement are addressed and then harmonic analysis is applied to check the impact of those anomalies in input measurement.

Effect of Timing Signal Time delay, discussed in the earlier section, impacts the angle of attack time history by causing a deviation from a single-frequency pure sinusoidal waveform (see Fig. 4). Harmonic analysis of the input is done to further investigate the timing issue.

Along with the two cases in Figs. 3 and 4, two other test cases used for this analysis were individual 
runs s738 and s815. One duty cycle slip was found in s738 while timing slips greater than 9 samples occurred more than 10 times in $\mathrm{s} 815$.

A model with the first harmonic is estimated and used to compute $R^{2}$ and $s^{2}$ from (10) and (7). Computed values are shown in the below table.

\begin{tabular}{|c|c|c||}
\hline Test Case & $\boldsymbol{R}^{2}$ & $\boldsymbol{s}^{2}$ \\
\hline $\mathrm{s} 726$ & 0.9986 & 0.0043 \\
\hline $\mathrm{s} 738$ & 0.9978 & 0.0143 \\
\hline $\mathrm{s} 768$ & 0.9954 & 0.0454 \\
\hline $\mathrm{s} 815$ & 0.9615 & 0.4478 \\
\hline
\end{tabular}

With bigger delays in time, $R^{2}$ value is decreasing and $s^{2}$ value is increasing. Though the trend makes sense, it is much less sensitive than expected for this analysis. This result brought attention to the difference in using actual measured time vs. a timing index for analysis. The timing index (sampling point number) can only be used with the assumption that test data are equally spaced in time. When timing delay is present, a legitimate option might be re-sampling to obtain data that is equally spaced in time. Computed values with resampled data are shown in the below table.

\begin{tabular}{||c|c|c||}
\hline \hline Test Case & $\boldsymbol{R}^{2}$ & $\boldsymbol{s}^{\mathbf{2}}$ \\
\hline $\mathrm{s} 726$ & 0.9986 & 0.0043 \\
\hline $\mathrm{s} 738$ & 0.9973 & 0.0203 \\
\hline $\mathrm{s} 768$ & 0.9850 & 0.1729 \\
\hline $\mathrm{s} 815$ & 0.1160 & 13.6544 \\
\hline
\end{tabular}

Due to a significant distortion in test case $\mathrm{s} 815, R^{2}$ is far less than 1 with a large error $\left(s^{2}\right)$ and implies that the model with the first harmonic is inadequate. For the other test cases, the values of $R^{2}$ and $s^{2}$ indicate much more adequate models have been estimated, although sensitivity to the timing errors are not as large as expected. Each individual run contained 10 oscillation cycles. By using 10 cycles for analysis the effect of the timing error was minimized and caused a lack of sensitivity to small time delays. Analysis with one cycle could show the sensitivity in $R^{2}$ value but the analysis with one cycle is not recommended for this study. So, except few extreme cases, analysis indicates the estimated models were adequate to explain the data $\left(R^{2} \sim\right.$ $\left.1, s^{2}<<1\right)$ and that the test data is reasonably well represented by linear models since a single-frequency harmonic was the dominant feature.

Calibration A second test is a comparison of the commanded and measured input signals without the hardware in the loop, i.e., without including the test rig dynamics.

A simplified block diagram of test setup is shown below and the output of the first conversion (from degree to voltage) is directly fed to the input of the second conversion (from voltage to degree) as depicted with a dashed line. With a proper conversion, commanded and measured input values should be the same.

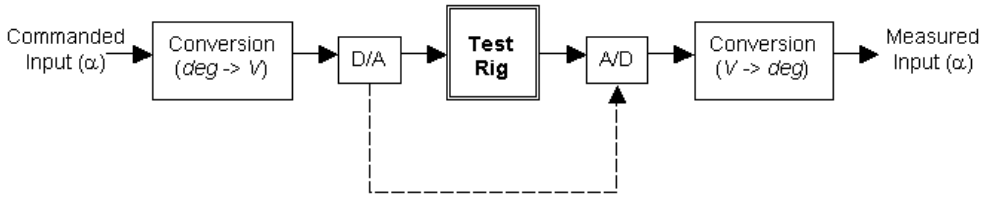

A test case is considered using $1.0 \mathrm{~Hz}$ forced oscillation data at $\alpha_{0}=70^{\circ}$. The top graph in Fig. 5 shows an example of commanded and measured angle of attack versus sample number index. The second plot shows the difference or residuals between these two graphs. Besides relatively large residual values, a bias error of $4.7^{\circ}$ exists and the amplitudes are different between the top and bottom of the oscillation $\left(6.2459^{\circ}\right.$ vs. $\left.6.5102^{\circ}\right)$. This is shown in the bottom two graphs by subtracting out the mean angle of attack from the top two graphs. This also removes the bias between the two plots. These new large residuals brought attention to a calibration technique used for this experiment. In this case, instead of an exact pointto-point table look up to convert degree to voltage and visa-versa, a $3^{\text {rd }}$ order polynomial approximation was used. This approach was intended to save test time but the cost was some distortion in the harmonic input signal. Deviation from a pure sinusoid introduced additional harmonics and biases into the experiment.

With the same test data, harmonic analysis with the first harmonic is used to compute $R^{2}$ and $s^{2}$ from (10) and (7). Computed values are presented in the below table.

\begin{tabular}{||c|c|c|c|}
\hline Mean $(\alpha)$ & Amplitude $(\alpha)$ & $\boldsymbol{R}^{2}$ & $\boldsymbol{s}^{\mathbf{2}}$ \\
\hline $74.7230^{\circ}$ & $6.3626^{\circ}$ & 0.9959 & 0.0023 \\
\hline
\end{tabular}

With the proper consideration of bias error (74.7230-70) and amplitude scaling error (6.3626/5), the test data presents reasonably linear dynamics. An adequate linear model was obtained with $R^{2}$ almost 1 and very small model variance.

Mechanical Issues The next test considers the mechanical system, Test Rig, in the above figure. A large "jump" distortion was found in several runs. As an example, Fig. 6 (s789: $\alpha_{0}=45^{\circ}$ and $f=1.5 \mathrm{~Hz}$ ) shows the $\alpha$ signal plotted against time in the top graph and then the same plot with a zoomed view of the distortion in the lower graph. In cases where this problem occurred the large jump in $\alpha$ was always followed by a large timing error. Jump errors were sometimes large enough to observe visually during the test. This seems likely to be caused by a mechanical system anomaly.

Another input anomaly that may be caused by the mechanical system is input saturation. Since input saturation can defeat the planned input spectrum used 
to excite a dynamic system, it can be an issue for the test engineer. Fig. 7 (s217: $0.5 \mathrm{~Hz}$ and $\alpha_{0}=5^{\circ}$ ) shows this issue for the single-frequency forced oscillation experiment. The effect of saturation is shown in the plots of $\alpha, C_{L}$, and $C_{N}$. From other test cases, saturation is observed in the low frequency experiments and does not appear to be affected by offset angle variation. This may imply a mechanical limitation of the test rig, however, the exact source of this error has not been identified.

To investigate the impact of mechanical issues on inputs, the same test data, shown in Fig. 6, is used for harmonic analysis. With only the first harmonic, computed values of $R^{2}$ and $s^{2}$ from (10) and (7) are shown in the below table. Since the jump anomaly was followed by a time delay, computed values with resampled data are also presented.

\begin{tabular}{||c|c|c|l||}
\hline Test Case & $\boldsymbol{R}^{\mathbf{2}}$ & $\boldsymbol{s}^{\mathbf{2}}$ & Note \\
\hline \multirow{3}{*}{ s789 } & 0.9926 & 0.0873 & with measured data \\
\cline { 2 - 4 } & 0.9484 & 0.6949 & with re-sampled data \\
\hline
\end{tabular}

For the analysis of input saturation, computed values of $R^{2}$ and $s^{2}$ from (10) and (7) for a test case $\mathrm{s} 217$ (Fig. 7 ) are shown in the below table. Similar to the last case, the model appears to be adequate.

\begin{tabular}{||c|c|c|c|}
\hline \hline Mean $(\alpha)$ & Amplitude $(\alpha)$ & $\boldsymbol{R}^{2}$ & $\boldsymbol{s}^{2}$ \\
\hline $5.3657^{\circ}$ & $5.3591^{\circ}$ & 0.9974 & 0.0110 \\
\hline
\end{tabular}

In this section, three issues were addressed and harmonic analysis has been applied to assess the impact by those issues. In general, the contribution from a large number of repeated cycles in each test case allows production of test data that is reasonably acceptable. However, these kinds of issues would need to be resolved for investigations into nonlinear regimes.

\section{Output Measurements}

After conversion to non-dimensional engineering units, aerodynamic total force and moment coefficients are direct output measurements. Substantial efforts have been made to develop methodology for ensuring data accuracy using various statistical measures. For this paper, analysis is done with the assumption that the test facility has followed recommended procedure ${ }^{6}$ and worked to obtain the accurate data. Even with this assumption, it is prudent for the investigator to evaluate experimental results. Fairly simple calculations can often be used to prevent a variety of errors and minimize data uncertainty.

In this section, a conventional linear aerodynamic model is assumed and harmonic analysis is performed on angle of attack and force and moment measurements to estimate the in-phase and out-of-phase model coefficients. Analysis of repeatability or dispersion of the time histories and the in-phase and out-of-phase components is investigated using the variance expressions given by (19) and (21).

Aerodynamic Force and Moment Coefficients

For the total force and moment outputs, mean and standard deviation of total aerodynamic loads are computed from the 10 repeated single runs. The variance at each time instant is used to compute overall variance. Then, harmonic analysis is applied to ensemble average run to estimate aerodynamic force coefficients with the parameter covariance matrix for the estimated coefficients.

A co-plot of 10 repeated runs is shown in Fig. 8. This case is for oscillations about $\alpha_{0}=20^{\circ}$ and at a frequency of $1.1 \mathrm{~Hz} . C_{N}$ measurement shows a good repeatability, while a degraded repeatability is depicted in $C_{m}$ measurement. This degradation is addressed in Ref. [1] and the cause is still unknown. A significant anomaly is found in $C_{A}$ measurement. It shows a large inconsistency over 10 repeated runs and the measurement was dominated by noise.

As a first test case, the first cycle of $C_{N}$ is used. A co-plot of 10 single runs and the corresponding plot of computed mean values with $2 \sigma$ bounds at each time instant are depicted in Fig. 9. Overall variance from (20)-(21) is

$$
s_{E}^{2}=\frac{1}{N} \sum_{i} s_{i}^{2}=0.9939 \times 10^{-4} .
$$

After using only the first harmonic for harmonic analysis in (2) to compute an estimate of $C_{N}$, a co-plot of measured $C_{N}$ and estimated $C_{N}$ are also shown in Fig. 9 (bottom plot). This figure illustrates the model with the first harmonic explains the measured data fairly well. Computed values of the parameter covariance from (7) for the estimated coefficients is

$$
s_{M}^{2}=\frac{1}{N} \sum_{i}\left[C_{N}(i)-\hat{C}_{N}(i)\right]^{2}=0.4224 \times 10^{-4} .
$$

In order to investigate other cases, the first cycle of $C_{m}$ from the same test case is used to compute the corresponding variances. Then, a different test case (1.1 Hz and $\alpha_{0}=60^{\circ}$ ) is used to compute variances for $C_{N}$ and $C_{m}$. Computed variances with the corresponding standard errors are presented in the below table.

\begin{tabular}{|c||l|l||l|l||}
\hline \multirow{2}{*}{} & \multicolumn{1}{c|}{$1.1 \mathrm{~Hz}$ and $\alpha_{0}=20^{\circ}$} & \multicolumn{2}{c|}{$1.1 \mathrm{~Hz}$ and $\alpha_{0}=60^{\circ} \mathrm{deg}$} \\
\cline { 2 - 5 } & \multicolumn{1}{c|}{$\boldsymbol{C}_{\boldsymbol{N}}$} & \multicolumn{1}{c|}{$\boldsymbol{C}_{\boldsymbol{m}}$} & $\boldsymbol{C}_{\boldsymbol{m}}$ \\
\hline \hline$s_{E}^{2}$ & $0.9939 \times 10^{-4}$ & $0.2462 \times 10^{-4}$ & $0.5859 \times 10^{-3}$ & $0.7201 \times 10^{-4}$ \\
\hline$s_{E}$ & 0.0100 & 0.0050 & 0.0242 & 0.0085 \\
\hline$s_{M}^{2}$ & $0.4224 \times 10^{-4}$ & $0.4487 \times 10^{-5}$ & $0.1376 \times 10^{-3}$ & $0.1628 \times 10^{-4}$ \\
\hline$s_{M}$ & 0.0065 & 0.0021 & 0.0117 & 0.0040 \\
\hline
\end{tabular}

$s_{E}^{2}$ is a computed variance from repeated runs and $s_{M}{ }^{2}$ is a computed one from model. Theoretically, the two variances are the same if there were no errors in 
modeling with the first harmonic and if accurate data with good repeatability were acquired during experiments. In these test cases, with $s_{E}{ }^{2}$ greater than $s_{M}{ }^{2}$, a larger error in test repeatability is implied relative to modeling error.

\section{In-phase and out-of-phase components}

Ensemble Average Runs The conventional procedure to obtain a model in forced-oscillation testing is to form an ensemble average of repeated runs. Harmonic analysis is then used to obtain the parameters in model equation (13). From (18) and (19), in-phase and out-ofphase components with the corresponding standard errors are computed. Results for normal force coefficient, $\mathrm{C}_{N}$, are presented in Tables 1 and plotted against the angle of attack in Fig. 10.

Ten Repeated Single Runs Harmonic analysis is also applied to individual runs. For $f=1.5 \mathrm{~Hz}$, Fig. 11 shows in-phase and out-of-phase components of normal force coefficient, with the $2 \sigma$ bounds, for each of the 10 runs against angle of attack. This figure provides repeatability and error bound information. Fairly larger deviations are shown in Fig. 11 while a good repeatability is observed for most test cases. In this case, a test engineer can quickly assess progress of the experiment and the presence of any outlier results.

Outlier Rejection Rules From Ref. [4], when the number of repeats $(N)$ is 10 , the ratio of maximum acceptable deviation to precision index is 1.96 with a corresponding probability of 0.95 . Applying Chauvenet's criterion ${ }^{4}$ to the test case shown in Fig. 11 $\left(f=1.5 \mathrm{~Hz}\right.$ and $\left.\alpha_{0}=55^{\circ}\right)$, the outlier screening metric $\left(1.96^{*} \sigma\right)$ requires the single run, $s 815$, to be rejected by this criterion. As described earlier, s815 has a significant timing delay. In-phase and out-of phase coefficients both for all 10 runs and for selected runs ( 9 runs in this case) are computed and shown in the table below. From the table, Chauvenet's criterion successfully removed the questionable test data and improved data consistency by reducing standard deviations.

\begin{tabular}{|l|c|c|c|c||}
\hline \hline \multirow{2}{*}{} & \multicolumn{2}{|c|}{ All 10 Runs } & \multicolumn{2}{c|}{ Selected Runs } \\
\cline { 2 - 5 } & Mean & $\sigma$ & Mean & $\sigma$ \\
\hline $\bar{C}_{N_{\alpha}}$ & 1.2603 & 0.0830 & 1.2406 & 0.0582 \\
\hline $\bar{C}_{N_{q}}$ & 4.8351 & 0.5424 & 4.9933 & 0.2224 \\
\hline
\end{tabular}

\section{Concluding Remarks}

NASA LaRC has been conducting a series of wind tunnel tests to develop and test mathematical models and system identification methodology for aircraft rigidbody aerodynamics in nonlinear unsteady flight regimes. Analysis of measurement accuracy, especially for nonlinear dynamic systems that may exhibit complicated behaviors, is an essential component of this ongoing effort. In this paper, tools for harmonic analysis of dynamic data and assessing measurement accuracy have been presented. A linear aerodynamic model is assumed that is appropriate for conventional forcedoscillation experiments, although more general models can be used with these tools. Application of the tools to experimental data is demonstrated and results indicate the levels of uncertainty in output measurements that can arise from experimental setup, calibration procedures, mechanical limitations, and input errors. During the evaluation, several issues have been highlighted: 1) duty-cycle slips in real-time experiments, 2) signal-to-noise ratio (SNR), 3) calibration, and 4) input design. Suggestions that could address these issues have been proposed for ensuring high measurement accuracy of future experiments.

Extension of this work may support implementation of closed-loop automated testing where feedback measurements are analyzed, in realtime, with the appropriate metrics to produce the highest data quality possible during dynamic testing. This would provide efficient experimental systems that can reduce experimental time and increase measurement accuracy.

\section{Acknowledgements}

Wind tunnel tests were carried out with the assistance of NASA researcher Jay M. Brandon and George Washington University student Leslie Gould.

\section{References}

1. Vladislav Klein, Patrick C. Murphy, Timothy J. Curry and Jay M. Brandon, "Analysis of Wind Tunnel Longitudinal Static and Oscillatory Data of the F-16XL Aircraft," NASA/TM-97-206276, December 1997.

2. Vladislav Klein and Patrick C. Murphy, "Estimation of Aircraft Nonlinear Unsteady Parameters From Wind Tunnel Data," NASA/TM-1998-208969, December 1998.

3. Patrick C. Murphy and Vladislav Klein, "Estimation of Aircraft Unsteady Aerodynamic Parameters From Dynamic Wind Tunnel Testing," AIAA Atmospheric Flight Mechanics Conference, Aug. 2001.

4. H. W. Coleman and W. Glenn Steele, Jr.: Experimentation and Uncertainty Analysis For Engineers, 1989.

5. Brandon, J. M.: Dynamic Stall Effects and Application to High Performance Aircraft. Special Course on Aircraft Dynamics at High Angles of Attack: Experiment and Modeling. AGARD Report No. 776, April 1991, pp. 2-1 to 2-15.

6. Michael J. Hemsch, "Development and Status of Data Quality Assurance Program at NASA Langley Research Center - Toward National Standards," AIAA Advanced Measurement and Ground Testing Technology Conference, June 1996. 


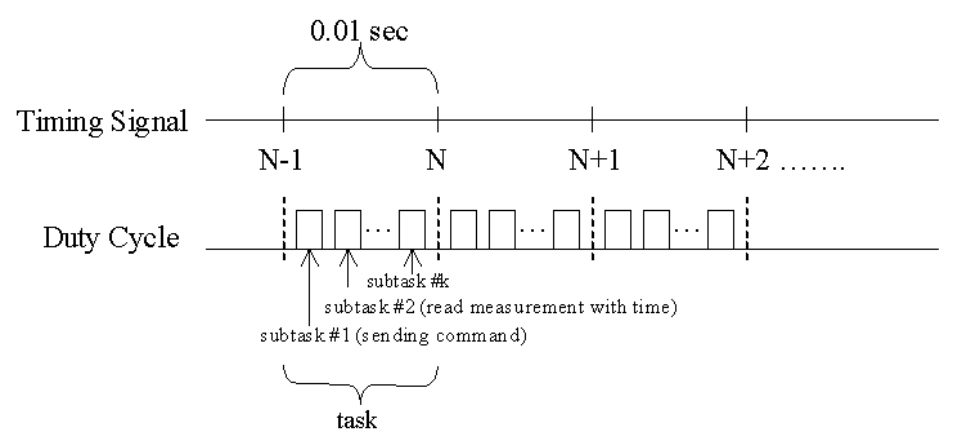

\begin{tabular}{|c|c|c|c|c|c|c|c|c|c|c|}
\hline 5.3857 & 2.5680 & 0.0043 & 2.5630 & 0.0054 & 2.5855 & 0.0057 & 2.4502 & 0.0106 & 2.5970 & 0.0063 \\
\hline 10.4589 & 2.8939 & 0.0034 & 2.8813 & 0.0046 & 2.8793 & 0.0046 & 2.7414 & 0.0109 & 2.8390 & 0.0053 \\
\hline 15.5519 & 2.9882 & 0.0027 & 2.9951 & 0.0041 & 2.9901 & 0.0037 & 2.8480 & 0.0110 & 2.9419 & 0.0049 \\
\hline 20.6637 & 3.0786 & 0.0036 & 3.1710 & 0.0051 & 3.1610 & 0.0045 & 3.0169 & 0.0118 & 3.1481 & 0.0059 \\
\hline 25.7697 & 2.5615 & 0.0083 & 2.9269 & 0.0073 & 3.0011 & 0.0070 & 2.9306 & 0.0120 & 3.1932 & 0.0079 \\
\hline 30.9134 & 1.7827 & 0.0047 & 2.4183 & 0.0061 & 2.5944 & 0.0057 & 2.5054 & 0.0126 & 2.9703 & 0.0086 \\
\hline 36.0349 & 1.2164 & 0.0055 & 2.0587 & 0.0074 & 2.1890 & 0.0071 & 2.0899 & 0.0127 & 2.5702 & 0.0080 \\
\hline 41.2319 & 0.9984 & 0.0066 & 1.7922 & 0.0063 & 1.8965 & 0.0053 & 1.7667 & 0.0123 & 2.2048 & 0.0076 \\
\hline 46.5557 & 0.8601 & 0.0035 & 1.4735 & 0.0052 & 1.5914 & 0.0066 & 1.5944 & 0.0102 & 1.9640 & 0.0082 \\
\hline 51.9286 & 0.7992 & 0.0042 & 1.3476 & 0.0068 & 1.3833 & 0.0056 & 1.4103 & 0.0076 & 1.7766 & 0.0086 \\
\hline 57.5852 & 0.7717 & 0.0046 & 1.1460 & 0.0056 & 1.2135 & 0.0060 & 1.2647 & 0.0099 & 1.6611 & 0.0161 \\
\hline 63.3500 & 0.7154 & 0.0044 & 0.9467 & 0.0077 & 1.0435 & 0.0060 & 1.0808 & 0.0102 & 1.4151 & 0.0238 \\
\hline
\end{tabular}

(a) In-phase component

\begin{tabular}{|c|c|c|c|c|c|c|c|c|c|c|}
\hline \multirow{2}{*}{$\alpha$ (deg.) $)$} & \multicolumn{2}{|c|}{$k=0.067$} & \multicolumn{2}{c|}{$k=0.121$} & \multicolumn{2}{c|}{$k=0.148$} & \multicolumn{2}{c|}{$k=0.201$} & \multicolumn{2}{c|}{$k=0.270$} \\
\cline { 2 - 11 } & $\bar{C}_{N_{q}}$ & $s\left(\bar{C}_{N_{q}}\right)$ & $\bar{C}_{N_{q}}$ & $s\left(\bar{C}_{N_{q}}\right)$ & $\bar{C}_{N_{q}}$ & $s\left(\bar{C}_{N_{q}}\right)$ & $\bar{C}_{N_{q}}$ & $s\left(\bar{C}_{N_{q}}\right)$ & $\bar{C}_{N_{q}}$ & $s\left(\bar{C}_{N_{q}}\right)$ \\
\hline 2.4036 & 1.7387 & 0.0627 & 2.0880 & 0.0447 & 2.3781 & 0.0399 & 2.3538 & 0.0260 & 2.3350 & 0.0236 \\
\hline 5.3857 & 1.6281 & 0.0650 & 1.9666 & 0.0450 & 2.2248 & 0.0390 & 4.3379 & 0.0533 & 2.2346 & 0.0236 \\
\hline 10.4589 & 1.6864 & 0.0510 & 0.4504 & 0.0383 & 2.1189 & 0.0314 & 4.5862 & 0.0553 & 2.3263 & 0.0198 \\
\hline 15.5519 & 1.6453 & 0.0412 & 0.2889 & 0.0344 & 2.0122 & 0.0255 & 4.5097 & 0.0556 & 2.0436 & 0.0185 \\
\hline 20.6637 & 3.3762 & 0.0546 & 1.2110 & 0.0420 & 2.7101 & 0.0308 & 4.8908 & 0.0591 & 2.1162 & 0.0222 \\
\hline 25.7697 & 8.6048 & 0.1242 & 5.5646 & 0.0606 & 5.7554 & 0.0480 & 6.7531 & 0.0601 & 3.2500 & 0.0296 \\
\hline 30.9134 & 17.2872 & 0.0696 & 10.4790 & 0.0505 & 9.3472 & 0.0389 & 8.6484 & 0.0628 & 4.6634 & 0.0321 \\
\hline 36.0349 & 25.8476 & 0.0824 & 13.2146 & 0.0613 & 10.9842 & 0.0480 & 9.3080 & 0.0635 & 5.5106 & 0.0297 \\
\hline 41.2319 & 28.3970 & 0.0979 & 12.2830 & 0.0525 & 10.2518 & 0.0358 & 8.3375 & 0.0618 & 5.1176 & 0.0285 \\
\hline 46.5557 & 17.3199 & 0.0516 & 9.8932 & 0.0431 & 8.4460 & 0.0447 & 7.1076 & 0.0511 & 4.2987 & 0.0306 \\
\hline 51.9286 & 11.5517 & 0.0622 & 6.3754 & 0.0565 & 5.9877 & 0.0380 & 5.6257 & 0.0378 & 3.4465 & 0.0320 \\
\hline 57.5852 & 7.7360 & 0.0688 & 5.4186 & 0.0460 & 5.1658 & 0.0405 & 4.8819 & 0.0493 & 2.8488 & 0.0597 \\
\hline 63.3500 & 7.3488 & 0.0648 & 4.6496 & 0.0631 & 3.9334 & 0.0406 & 3.9036 & 0.0509 & 2.1453 & 0.0881 \\
\hline
\end{tabular}

\section{(b) Out-of-phase component}

Table 1. In-phase and out-of-phase components of $C_{N}$ coefficient.

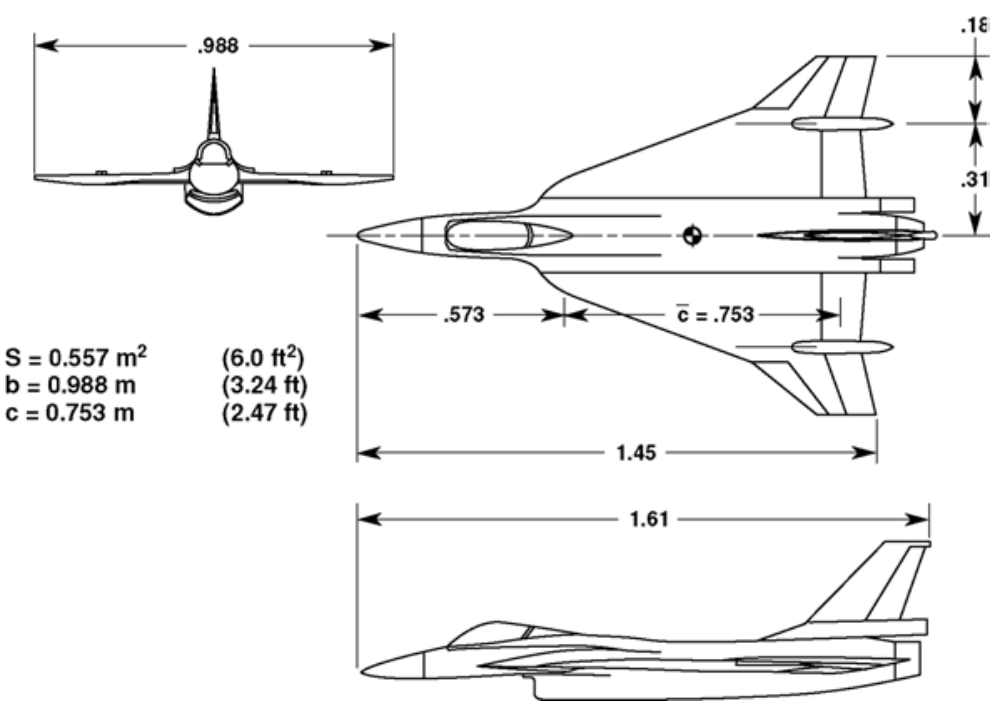

Figure 1. Three-dimensional view of 10\% F-16 XL model.
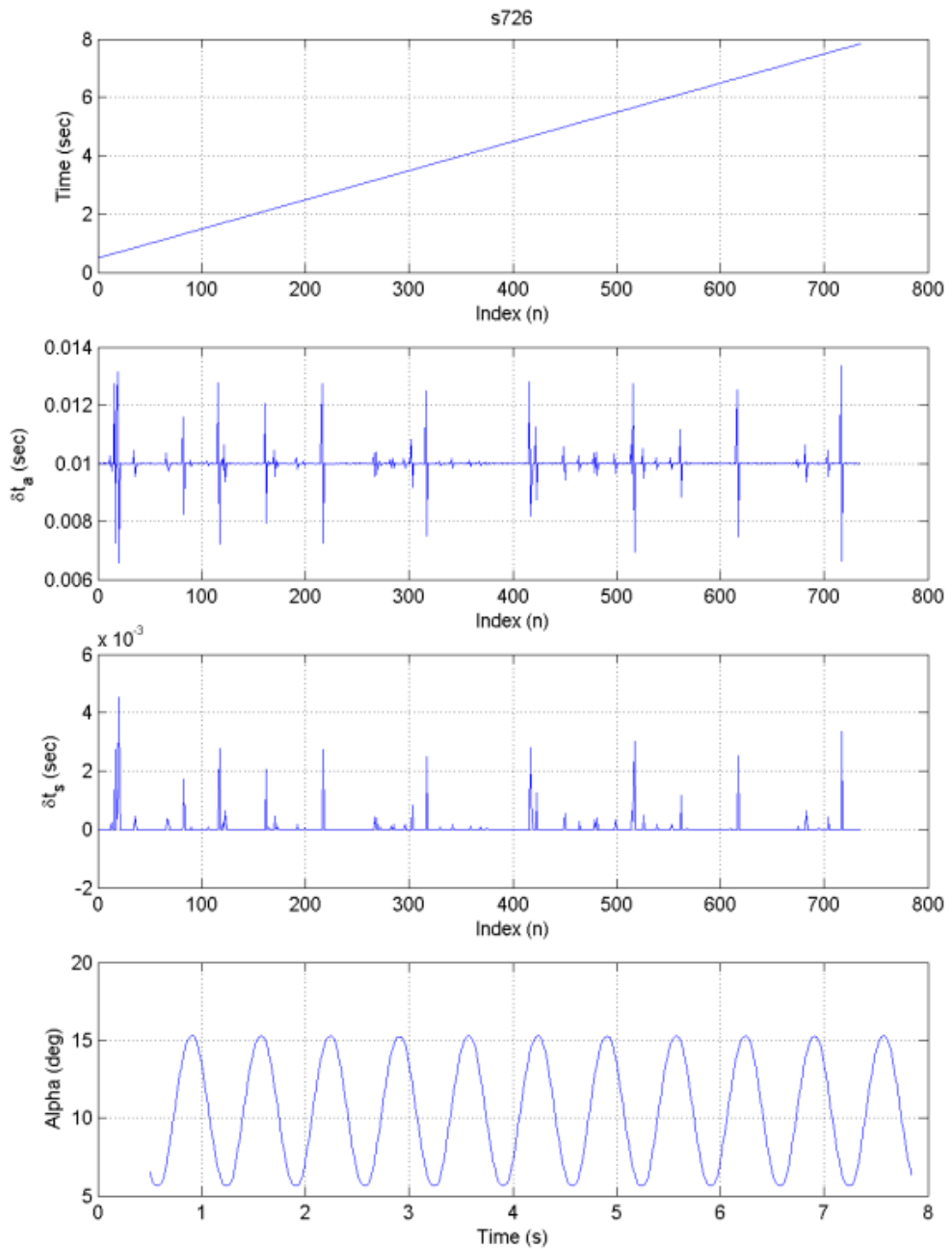

Figure 3. Typical timing signal of dynamic test data. 

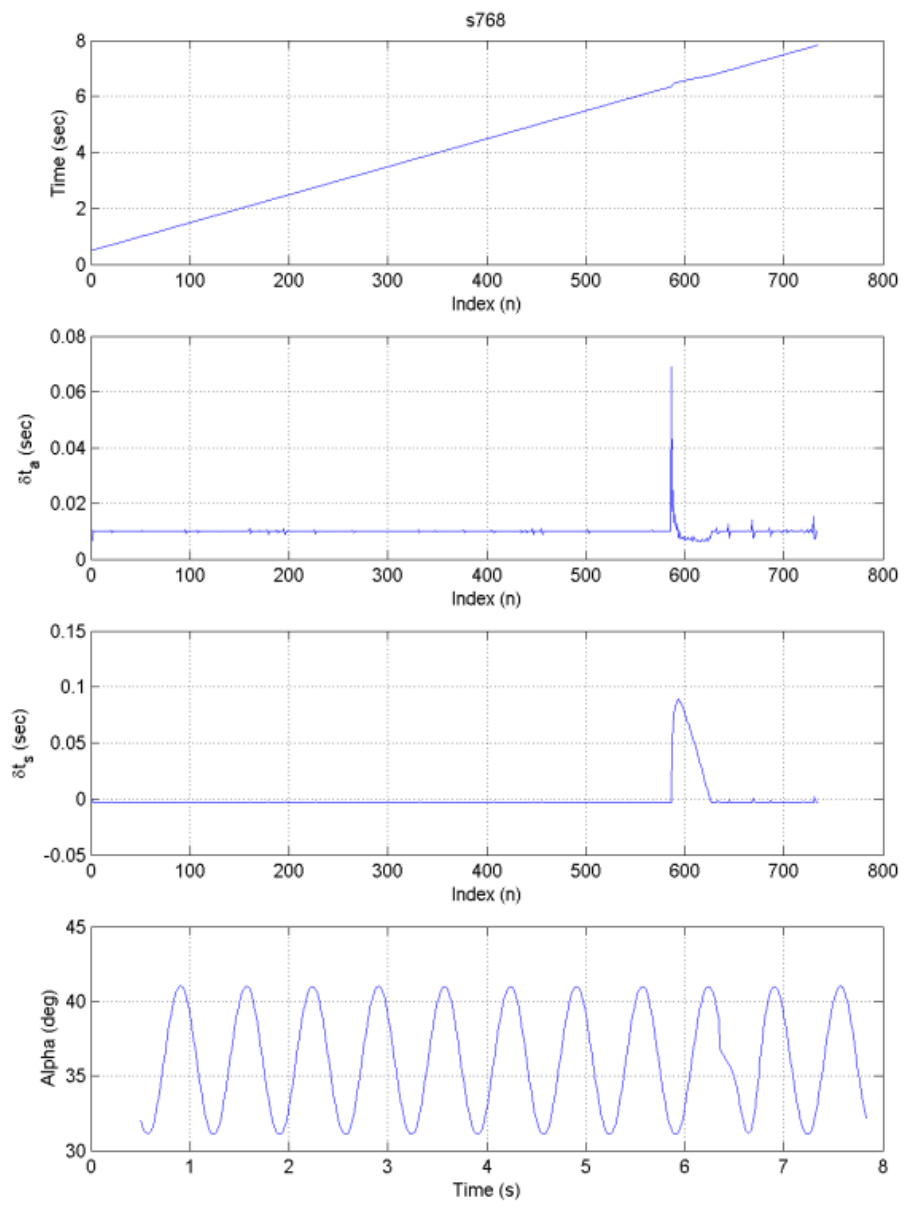

Figure 4. Dynamic test data with the slippage of duty cycles.
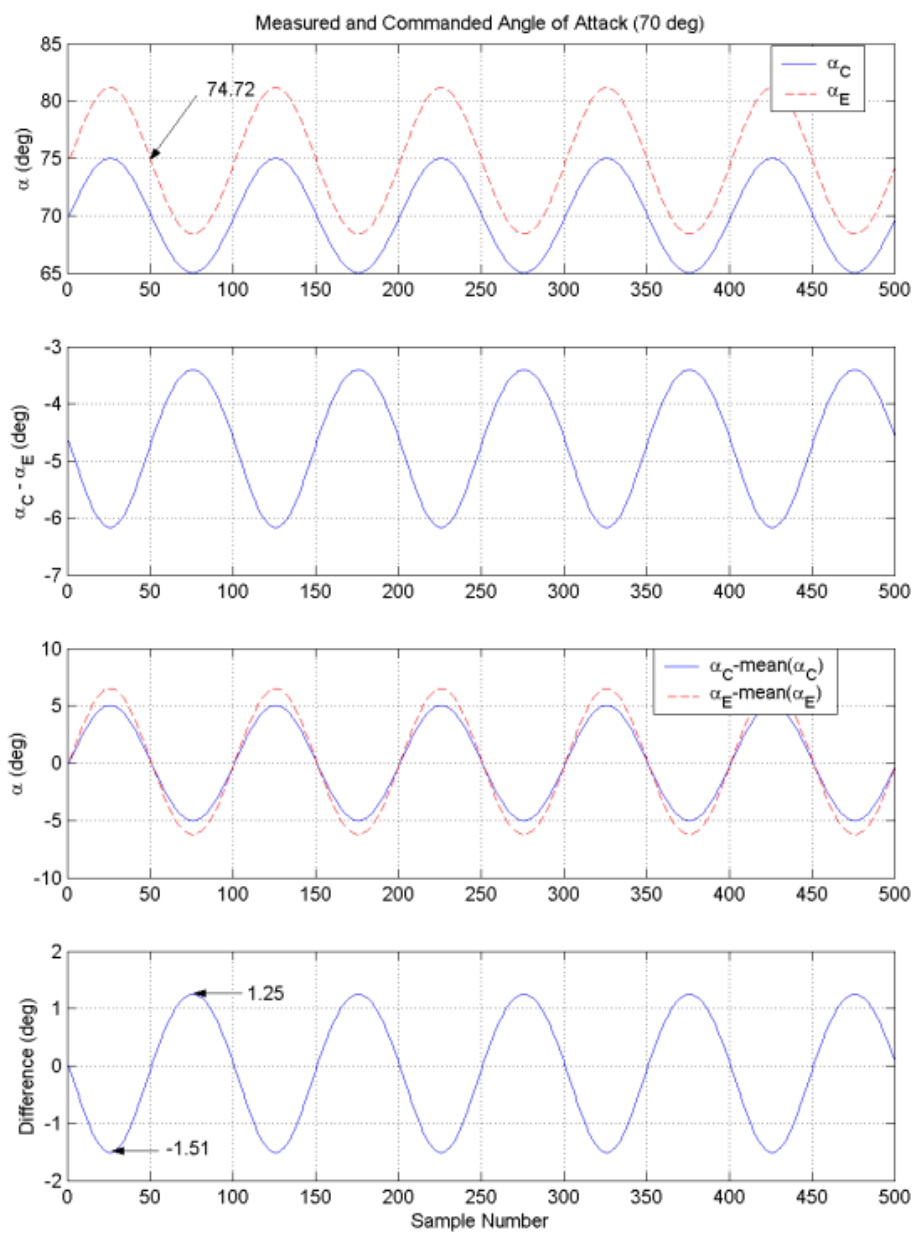

Figure 5. Measured and Commanded Angles of Attack.
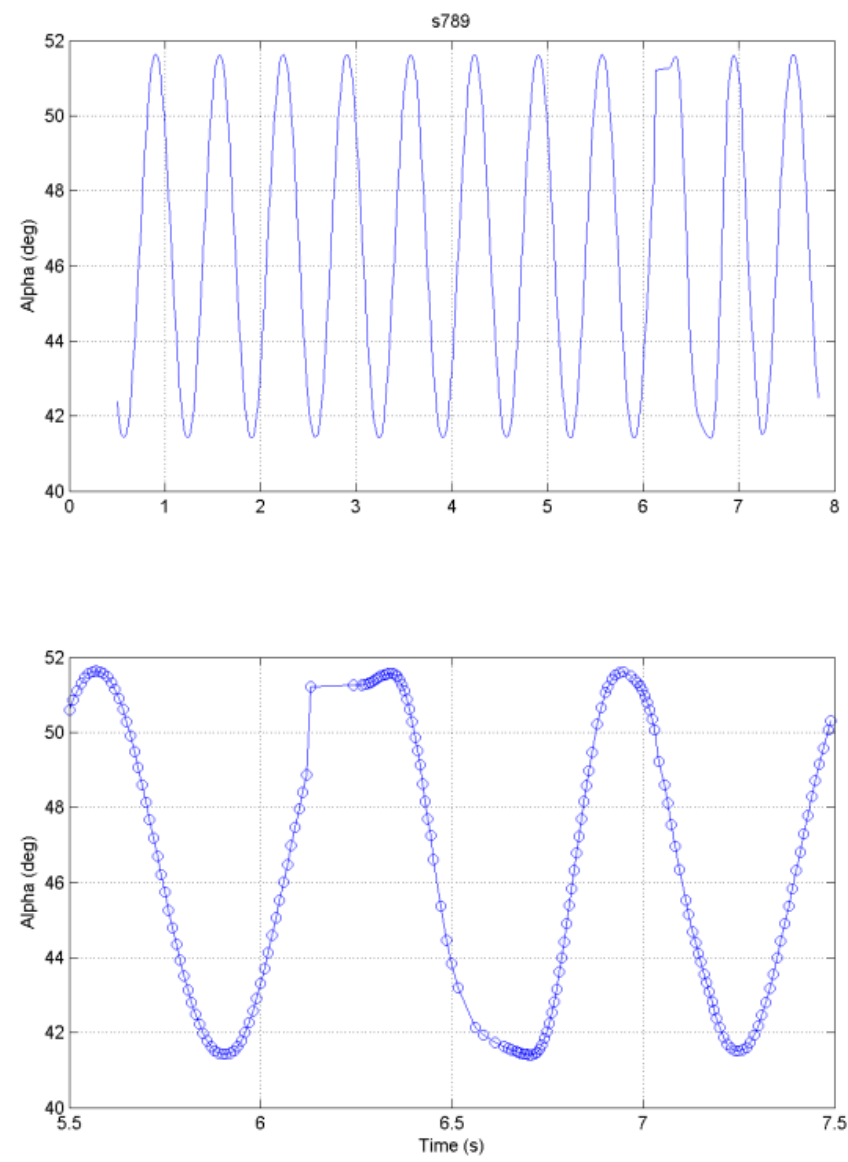

Figure 6. Jump in Angle of Attack Time History.
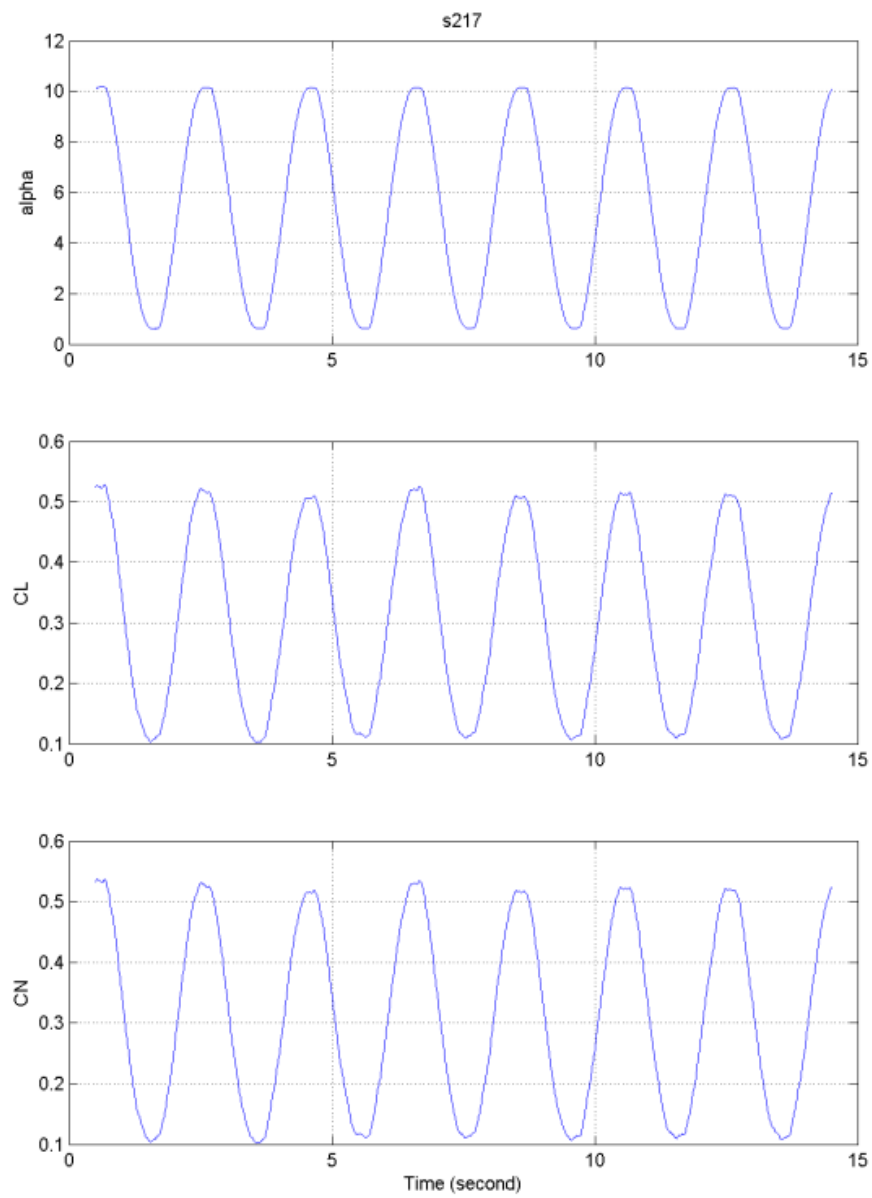

Figure 7. Saturated Input Command and Corresponding $C_{L}$ and $C_{N}$ Time Histories. 

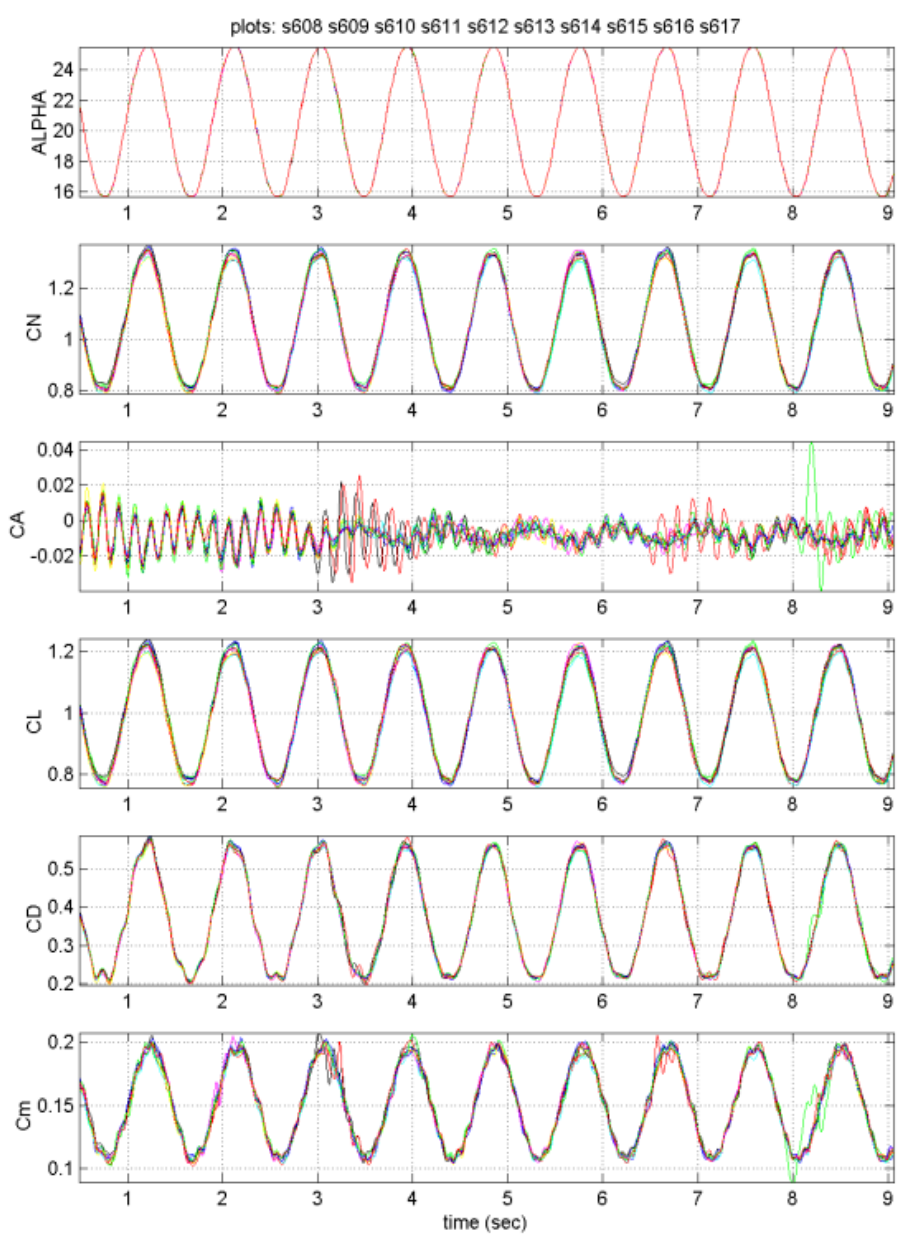

Figure 8. Typical dynamic test data: Ten repeated measurements.
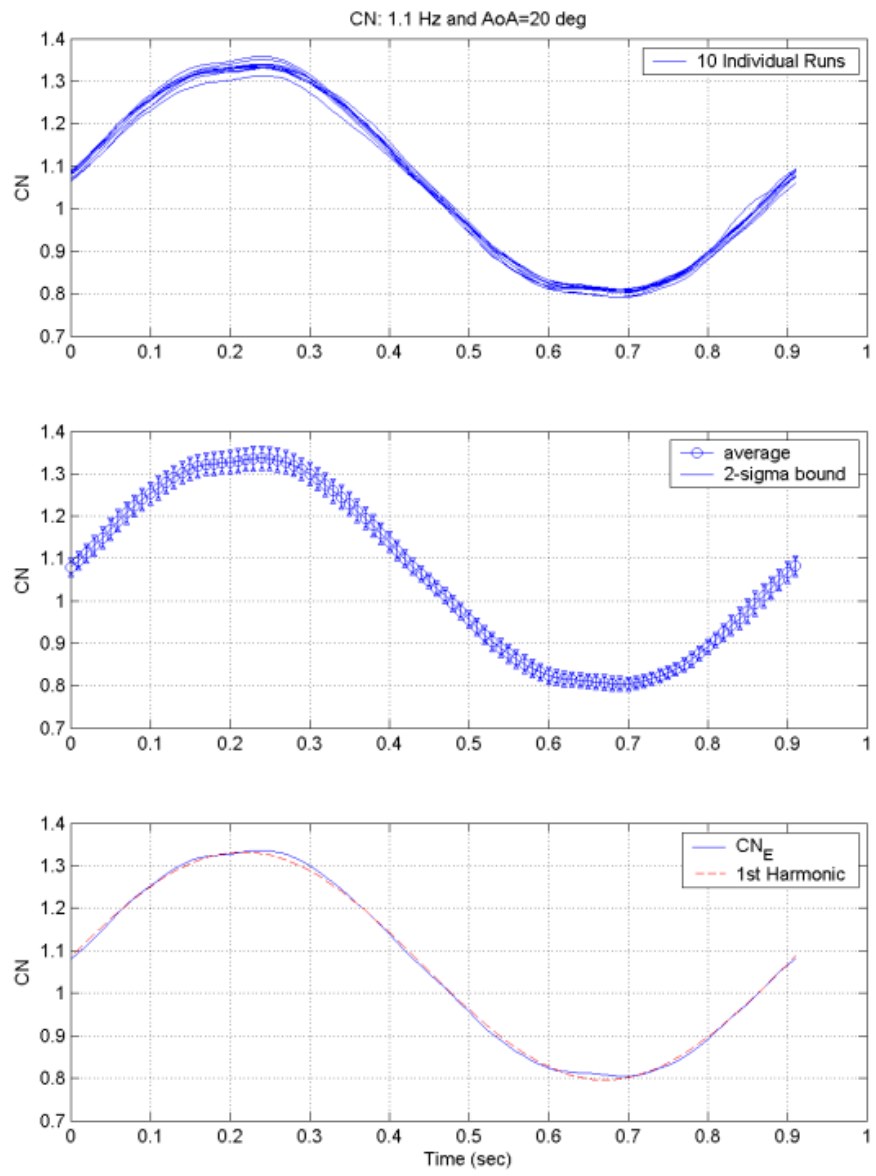

Figure 9. Co-plot of 10 Runs, Mean Values with $2 \sigma$ bound, and Co-plot of Measured $C_{N}$ and Estimated $C_{N}$.
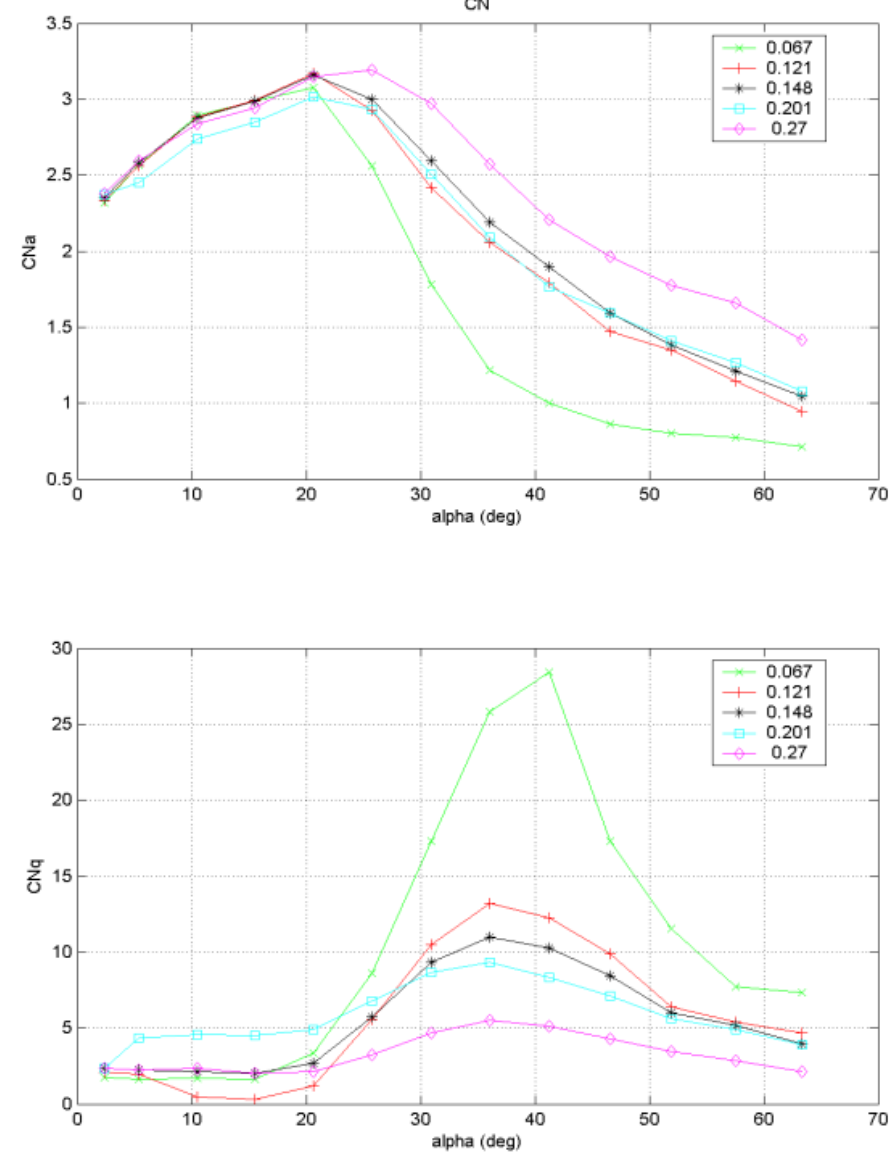

Figure 10. Variation of in-phase and out-of-phase components of $C_{N}$.
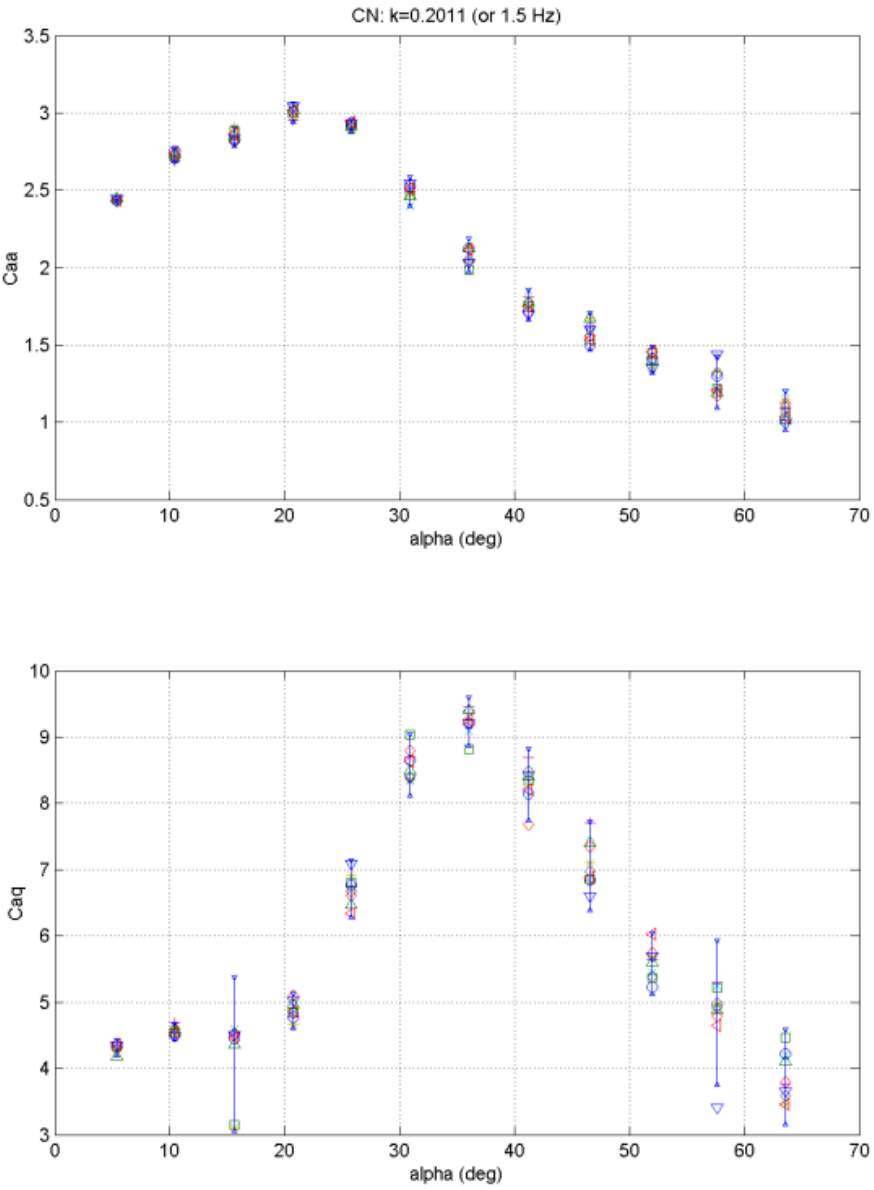

Figure 11. Co-plot of 10 single-runs with $2 \sigma$ bounds of in-phase and out-of-phase components of $C_{N}$ for $f=1.5 \mathrm{~Hz}$. 